\title{
Mühsam ernährt sich das Eichhörnchen
}

\author{
Um die erfolgreiche Implementierung der Checkliste im Spital zu fördern, hat die \\ Stiftung für Patientensicherheit im Rahmen der nationalen Qualitätsstrategie des \\ Bundes das Pilotprogramm «progress! Sichere Chirurgie» lanciert.
}

\section{Anna Mascherek ${ }^{a}$, \\ Paula Bezzola ${ }^{b}$ \\ David Schwappach ${ }^{c}$}

a Wissenschaftliche Mitarbeiterin Stiftung für Patientensicherheit

b Stv. Geschäftsführerin Stiftung für Patientensicherheit

c Wissenschaftlicher Leiter Stiftung für Patientensicherheit
Korrespondenz:

Paula Bezzola

Stiftung für Patientensicherheit Asylstrasse 77

CH-8032 Zürich

Tel. 0432441480

Fax 0432441481

info[at]patientensicherheit.ch
Das Thema Patientensicherheit ist spätestens seit dem Bericht «To Err is Human» [1] der Institutes of Medicine im Jahr 2000 im öffentlichen, wissenschaftlichen und professionellen Diskurs fest etabliert. Viele Anstrengungen und Kampagnen sind in der Praxis durch- und eingeführt worden, und auch die Wissenschaft hat sich mit der Erforschung wirksamer Strategien und Interventionen beschäftigt [2] In einem aktuellen Artikel in den Annals of Internal Medicine haben führende Wissenschaftler aus dem Bereich der Patientensicherheit eine systematische Evaluation aktuell bestehender Strategien zur Verbesserung der Patientensicherheit vorgenommen [3]. Basierend auf der Bewertung von vorhandener empirischer Evidenz für die Wirksamkeit und die Umsetzbarkeit der Intervention, erstellte die Expertengruppe um Paul Shekelle eine Liste mit den Top-10-Strategien für mehr Patientensicherheit. Diese zehn werden von den Wissenschaftlern als unbedingt empfehlenswert für die Implementierung im Betrieb eingestuft [3]. In dem Artikel wird deutlich, dass Massnahmen bekannt und gute Interventionen entwickelt worden sind, die einen erheblichen Effekt auf die Sicherheit der Patienten und die Vermeidung unerwünschter Ereignisse haben. Grossangelegte Interventionen, die durch wissenschaftliche Evaluation begleitet wurden, finden sich im Versorgungsalltag bereits wieder, so zum Beispiel Massnahmen zur Förderung der Händehygiene [4]. Manche Interventionen haben sogar Eingang in nationale Empfehlungen gefunden. So gilt die Verwendung der chirurgischen Checkliste der WHO in England seit 2009 und in Frankreich seit 2010 als nationaler Standard für Gesundheitseinrichtungen $[5,6]$.

Neben diesen erfreulichen Entwicklungen weisen die Autoren der Studie jedoch auch kritisch darauf hin, dass die Umsetzung vor Ort trotz allem oft nicht komplikationslos und vor allem nicht konsequent erfolgt. Die Diskrepanz zwischen dem, was möglich wäre, und dem, was tatsächlich umgesetzt wird, ist gross. Bereits an anderer Stelle haben Schwappach und Conen darauf hingewiesen und mögliche Ursachen benannt [7]. Oftmals werden selbst vergleichsweise einfache und kostengünstige Massnahmen wie beispielsweise die Händehygiene und die chirurgische Checkliste weder flächendeckend noch konsequent angewandt. Die Umsetzung solcher Interventionen verlangt das Engagement ganzer Organisationen. Deswegen geschieht sie meist zu langsam und zu unvollständig [7]. Nationale wie internationale Bestrebungen können diesen Prozess bestärken [8]. Auch die Verhaltensänderung jeder einzelnen Person spielt eine wichtige Rolle bei der vollständigen und dauerhaften Umsetzung. Mangelndes Wissen oder geringes Problembewusstsein sind oftmals Hinderungsgründe [7]. Vor allem aber wird Engagement für Patientensicherheit noch von zu vielen Fachpersonen als etwas «Zusätzliches» verstanden, das nicht Kernbestandteil der klinischen Tätigkeit ist. Ein prominentes Beispiel für die Diskrepanz zwischen wissenschaftlicher Evidenz und flächendeckender, vollständiger Umsetzung in der Praxis ist der Einsatz der chirurgischen Checkliste.

\section{WHO-Checkliste als wichtige Sicherheits- massnahme}

Seit der weltweiten Lancierung der chirurgischen Checkliste der WHO 2009 ist ihre Wirksamkeit als Instrument zur Förderung der Patientensicherheit in verschiedenen Settings vielfach gezeigt worden [9, 10]. Mit der systematischen Anwendung der chirurgischen Checkliste lassen sich unerwünschte Ereignisse verhindern oder rechtzeitig auffangen. Die chirurgische Checkliste erhöht die Patientensicherheit, indem sie die konsequente Einhaltung von Standards fördert (z.B. Markierung der Operationsstelle, rechtzeitige Antibiotikaprophylaxe) und indem sie die hierarchiebedingt gehemmte Kommunikation im interprofessionellen Team verbessert [1113]. Die Wirksamkeit der Checkliste zur Förderung der Patientensicherheit ist evident, und die Implementierung somit unbedingt empfehlenswert (siehe auch [3]). Jedoch zeigen neuere Studien, dass auch bei einer so substantiell wirksamen und vergleichsweise einfachen Intervention die Vorgabe zur Anwendung in den Spitälern allein nicht ausreicht, um die Patientensicherheit massgeblich zu verbessern $[14,15]$. Die Checkliste ist nur wirksam, wenn sie immer und zu 100\% richtig angewendet wird. Patienten können von der Checkliste nicht profitieren, wenn diese nur bei einigen Patienten, nur in Teilen oder falsch eingesetzt wird. 


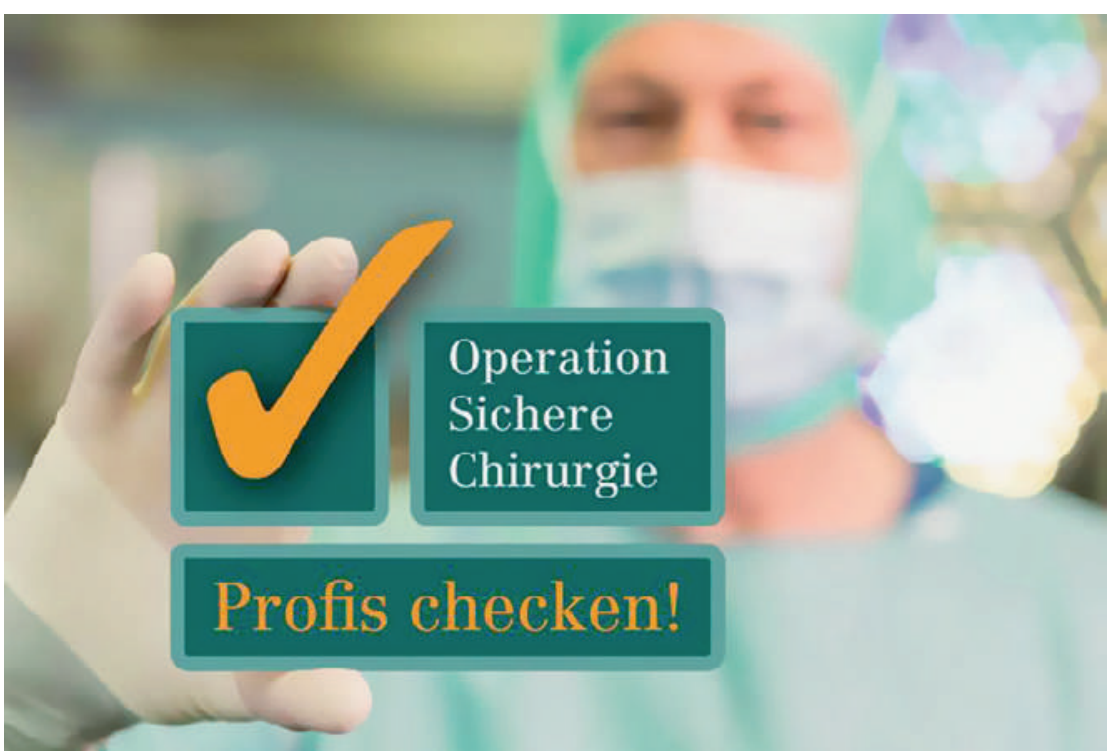

Wichtige Erfolgsfaktoren für die Wirksamkeit der chirurgischen Checkliste: hohe Compliance, gute Kommunikation im Team, Anpassung an spezifische Gegebenheiten des Spitals.

In einem systematischen Literaturreview zeigen Borchard und andere [11], dass wesentliche Erfolgsfaktoren für die Wirksamkeit der chirurgischen Checkliste eine hohe Compliance, gute Kommunikation im Team und die Anpassung der Checkliste an die Gegebenheiten des spezifischen Spitals sind. Nur dann lassen sich signifikante Verbesserungen im klinischen Outcome nachweisen.

Ursachen für die nicht korrekte oder unvollständige Anwendung der Checkliste sind oftmals Zeitdruck und die klinische Routine. Weitere Aspekte, die die richtige Anwendung und damit die Wirksamkeit der Checkliste verhindern, sind mangelndes Wis-

\section{Realität in Schweizer Betrieben}

Die Frage nach dem Stand der konkreten Implementierung und deren Erfolg stellt sich auch konkret für die Schweiz. Im Dezember 2012 hat deswegen die Stiftung für Patientensicherheit eine Studie zu Anwendung, zum Wissen und zu Einstellungen gegenüber chirurgischen Checklisten durchgeführt. Ungefähr 6000 Chirurgen, Anästhesisten und leitendes OP-Personal und Anästhesiepflege wurden schweizweit befragt. Die Antworten der knapp 1400 Teilnehmenden zeigen, was bereits erreicht wurde, machen aber auch den Optimierungsbedarf deutlich.

So nutzt nur jede dritte Fachperson alle drei Teile der WHO-Checkliste. Checks vor ( «Sign In») und nach der Operation ( «Sign Out») werden oft vernachlässigt. Die meisten Personen (60 Prozent der Ärzte, 80 Prozent des OP-Personals und der Anästhesiepflege) arbeiten nur mit dem Mittelteil («Team Time Out»). Das heisst, dass selbst in Betrieben, in denen die Checkliste eingesetzt wird, oft keine vollständige Umsetzung der empfohlenen Intervention stattfindet, was eine reduzierte Wirksamkeit zur Folge hat [11, 12, 14, 15]. Aus der Befragung wird auch deutlich, dass die Wissensvermittlung in vielen Betrieben zu kurz gekommen ist, auch wenn die allgemeine Wahrnehmung der Checkliste als wichtiges Tool für die Patientensicherheit bei der Mehrheit etabliert ist. So erachten 90 Prozent der Fachpersonen die chirurgische Checkliste als wichtiges Instrument zur Förderung der Patientensicherheit. Und die überwiegende Mehrheit (87 Prozent) der Fachpersonen möchte, dass die WHO-Checkliste angewendet wird, falls sie selber operiert würden. Trotzdem konnte nur eine von vier Fachpersonen über 80 Prozent der Wissensfragen des Fragebogens zur WHO-Checkliste richtig beantwor-

\section{Oft findet keine vollständige Umsetzung der empfohlenen Intervention statt, was eine reduzierte Wirksamkeit zur Folge hat.}

sen über das «Wie» und «Warum» bei den Beteiligten, sowie Schwierigkeiten in der Teamkommunikation und hierarchiebedingte Hindernisse [13, 14]. Aber auch mangelndes Problembewusstsein, bezogen auf die Notwendigkeit, spezifische und ganz allgemeine Massnahmen zur Verbesserung der Patientensicherheit einzuführen, kann hinderlich sein [7]. Hinzu kommt, dass die Anwendung der Checkliste nicht nur von einzelnen Personen abhängt, sondern systematisch und systemisch auch von Führungspersonen als Sicherheitsstandard im OP-Alltag verankert werden muss. Zusätzlich muss die Checkliste an lokale Gegebenheiten angepasst werden. Nur so kann die Checkliste langfristig und nachhaltig als elementare Sicherheitsmassnahme etabliert und zur Selbstverständlichkeit werden. ten. Ein weiterer wichtiger Punkt, bei dem auch in der Schweiz Optimierungsbedarf besteht, ist die systemische, gesamtbetriebliche Unterstützung und Förderung der Checkliste. Insgesamt haben 6 von 10 Personen das Gefühl, keinen Einfluss auf die Entscheidung zu haben, ob die Checkliste verwendet wird. Hier wird deutlich, dass die Anwendung von Checklisten eine gesamtbetriebliche Aufgabe darstellt, die von Einzelpersonen weder im positiven noch im negativen Sinn entschieden werden kann.

\section{Nationales Pilotprogramm der Stiftung für Patientensicherheit}

Um die empirisch abgesicherten Wirkfaktoren für eine erfolgreiche Implementierung der Checkliste im Spital spezifisch zu fördern und dadurch deren 


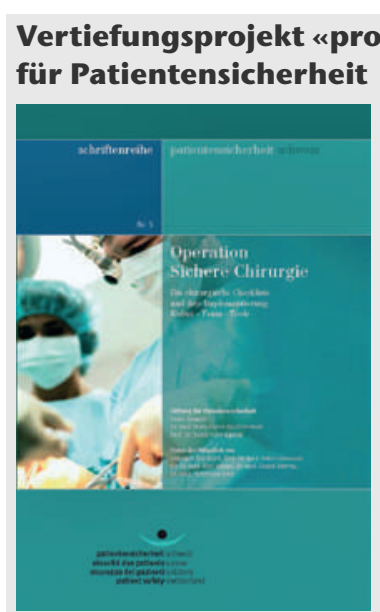

Das Pilotprogramm läuft unter dem Slogan «Operation Sichere Chirurgie - Profis checken!». Die Grundlagen wurden in enger Kooperation mit zahlreichen Experten aus dem Schweizer Gesundheitswesen und den Fachgesellschaften der fmCh sowie den Berufsverbänden des OP-Personals und der Anästhesiepflege erarbeitet. Das Programm wurde von der Stiftung für Patientensicherheit entwickelt und wird von ihr umgesetzt. Es ist gleichzeitig Bestandteil der Qualitätsstrategie des Bundes im schweizerischen Gesundheitswesen und wird massgeblich vom Bundesamt für Gesundheit BAG finanziert. Inhaltliche Basis bildet die Schrift «Operation Sichere Chirurgie», Stiftung für Patientensicherheit (ISBN 3-905803-09-7).

Kontakt: Stiftung für Patientensicherheit, Paula Bezzola, MPH, Tel. 04324414 80, bezzola[at] patientensicherheit.ch

Interessierte Betriebe können sich bis 3. Juni 2013 anmelden auf www.patientensicherheit.ch

korrekte und flächendeckende Anwendung in der Schweiz weiter voranzutreiben, hat die Stiftung für Patientensicherheit im Rahmen der nationalen Qualitätsstrategie des Bundes ein nationales Pilotprogramm «progress! Sichere Chirurgie» lanciert. Ziel des Programms ist die ausnahmslose und korrekte Anwendung der Checkliste, um so die Zahl der unerwünschten Ereignisse in der invasiven Medizin zu senken. Teil des Programms ist ein zweijähriges Vertiefungsprogramm mit Pilotbetrieben, das sich an Betriebe mit interprofessionellen Projektteams (Chirurgie, Anästhesie, OP-Pflege, QM und Klinikleitung) richtet, die zielorientiert, in gemeinschaftlichem Vorgehen, Verbesserungen bei der korrekten Anwendung der Checkliste erreichen wollen (Details siehe Kasten). Die Vernetzung, die Wissensvermittlung und die Erhöhung der Compliance werden konkret unterstützt. Einen zentralen Punkt des Pilotprogramms stellt zudem die wissenschaftlich fundierte Evaluation der wichtigsten Prozesse für eine erfolgreiche Implementierung der Checkliste dar. Die Wissensvermittlung, das Sicherheitsklima, Häufigkeit und Qualität der Anwendung, werden im Rahmen von «progress! Sichere Chirurgie» zu mehreren Zeitpunkten evaluiert. Die vorhandenen Ressourcen sollen so auf die Verbesserung derjenigen Prozesse verwendet werden, die für die erfolgreiche Implementierung und damit für die Wirksamkeit der Checkliste ausschlaggebend sind. Durch die gezielte Unterstützung und Begleitung der Betriebe soll erreicht werden, dass nach Abschluss des Programms die Checkliste konsequent und richtig eingeführt ist und somit ein weiterer Schritt in Richtung mehr Patientensicherheit und weniger unerwünschte Ereignisse getan worden ist.

\section{Literatur}

1 Institute of Medicine. To err is human. Building a safer health system. Washington, DC: National Academy Press; 2000.

2 Shekelle P, Wachter R, Pronovost P, Schoelles K, McDonald KM, Dy S, et al. Making Health Care Safer II: An updated critical analysis of the evidence for patient safety practices. Comparative Effectiveness Review No. 211. Rockville, MD; 2013. Report No.: AHRQ Publication No. 13-E001-EF.

3 Shekelle PG, Pronovost PJ, Wachter RM, McDonald KM, Schoelles K, Dy SM, et al. The Top Patient Safety Strategies That Can Be Encouraged for Adoption Now. Ann Intern Med 2013 Mar 5;158(5_Part_2):365-8.

4 Sax H, Allegranzi B, Uckay I, Larson E, Boyce J, Pittet D. «My five moments for hand hygiene»: a user-centred design approach to understand, train, monitor and report hand hygiene. The Journal of hospital infection 2007 Sep;67(1):9-21.

5 National Health Service. WHO Surgical Safety Checklist. www nrls npsa nhs uk/resources/ ?EntryId45=59860 2009 September 20 (cited 2013 Mar 8).

6 Haute Autorité de Santé. Pour une meilleure utilisation de la check-list sécurité du patient au bloc opératoire. www has-sante fr/portail/jcms/c_1173830/pour-unemeilleure-utilisation-de-la-check-list-securite-dupatient-au-bloc-operatoire 2013 (cited 2013 Mar 8)

7 Schwappach DL, Conen D. Patient safety - who cares? Swiss Med Weekly. 2012; Jul 16;142:w13634.

8 Pronovost P, Needham D, Berenholtz S, Sinopoli D, Chu $\mathrm{H}$, Cosgrove $\mathrm{S}$, et al. An Intervention to Decrease Catheter-Related Bloodstream Infections in the ICU. N Engl J Med. 2006; Dec 28;355(26):2725-32.

9 Haynes AB, Weiser TG, Berry WR, Lipsitz SR, Breizat AH, Dellinger EP, et al. A Surgical Safety Checklist to Reduce Morbidity and Mortality in a Global Population. N Engl J Med. 2009; Jan 14;NEJMsa0810119.

10 World Health Organization. Checklist implementation manual 2009, Safe surgery saves lives. www.who.int/patientsafety/safesurgery/tools_ resources/en/index html 2009 (cited 2013 Mar 8).

11 Borchard A, Schwappach DLB, Barbir A, Bezzola PA Systematic Review of the Effectiveness, Compliance, and Critical Factors for Implementation of Safety Checklists in Surgery. Ann Surg. 2012;256(6):925-33.

12 van Klei WA, Hoff RG, van Aarnhem EE, Simmermacher RK, Regli LP, Kappen TH, et al. Effects of the introduction of the WHO «Surgical Safety Checklist» on in-hospital mortality: a cohort study. Ann Surg. 2012; Jan;255(1):44-9.

13 Fourcade A, Blache JL, Grenier C, Bourgain JL, Minvielle E. Barriers to staff adoption of a surgical safety checklist. BMJ Qual Saf. 2012; Mar;21(3):191-7.

14 Vats A, Vincent CA, Nagpal K, Davies RW, Darzi A, Moorthy K. Practical challenges of introducing WHO surgical checklist: UK pilot experience. BMJ. 2010; Jan 13;340(jan13_2):b5433.

15 Bliss LA, Ross-Richardson CB, Sanzari LJ, Shapiro DS, Lukianoff AE, Bernstein BA, et al. Thirty-Day Outcomes Support Implementation of a Surgical Safety Checklist. J Am Coll Surg. 2012;Dec;215(6):766-76. 